\title{
Research on the Floating Characteristic of Burnishing Heads of Magnetic Disks*
}

\author{
Wang XIAN-KUI, ${ }^{* *}$ Duan GUANG-HONG, ${ }^{* *}$ \\ Lei YUAN-ZHON** and Bai YONG**
}

\begin{abstract}
There are some projections on a surface of a magnetic disk after polishing. These projections influence the flight stability of the magnetic head. This paper describes a simple approximate method to calculate the static floating characteristic of the burnishing head. The difference between the calculated results and the experimental data is small. It is successful to design the burnishing head in the production of magnetic disk. The result experimental shows that the burnishing head can fly across the magnetic disk surface with $0.2 \mu \mathrm{m}$ height and remove projections on it.
\end{abstract}

Key Words : Projection, Burnishing Head, Magnetic Disk

\section{Introduction}

In order to increase the storage density of the magnetic recording devices, the flying spacing (height) between flying head and disk surface should be reduced as small as possible. The space between the magnetic flying head (slider) and the rotating disk is created by aerodynamics action. To obtain a small and stable spacing, the magnetic flying head should follow the disk surface closely and the magnetic disk surface should have a very small of value roughness, as well as planeness. At the same time, the height of the projections on the coated disk surface should be limited. Otherwise head-disk crashes will occur and damage the disk device. The finishing surface of the high density disk is obtained by polishing, followed by in technical treatment which is called burnishing.

Burnishing technique is a method of removing the projections from the disk surface. A special flying

* Received 9th October, 1989

- National Natural Science Foundation of China, Beijing, P. R. China

** Department of Precision Instruments \& Mechanology, Tsinghua University, Beijing, P. R. China head made of a hard material (blue stone, sapphire), which is called burnishing head, flies above a rotating disk with a very small spacing. It touches the projections on the disk surface repeatedly. After a number of contacts, the projections can be eliminated, shown in Fig. 1.

In our apparatus, with an acoustic emission (AE) detection system, the positions of projections on the disk surface can be artificial out. The whole burnishing process can be controlled automatically by microcomputer and the results of the detection can be recorded and printed out.

There are many key techniques in development of the apparatus. One of them is burnishing. In the burnishing process, the burnishing head touches the

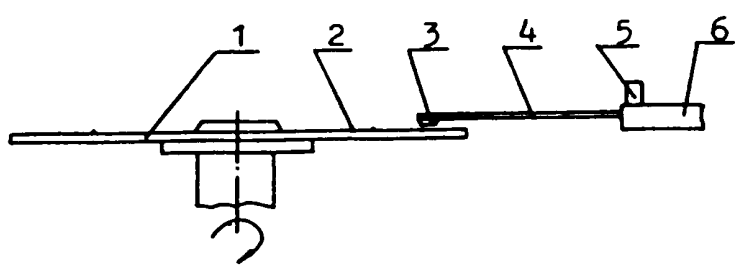

Fig. 1 Projection burnishing equipment 1-magnetic disk 4-arm of burnishing head 2-projection 5-AE transducer 3-burnishing head 6-fixed base 
projections repeatedly with a very small flying spacing. Excellent flying characters are needed. The flying spacing determines the maximum height of the projections on the disk surface after burnishing. So it is very important to choose the correct structure and dimensional parameters for the burnishing head to obtain the desired flying spacing.

The burnishing head developed is made of artificial sapphire with a high hardness. It is not easy to machine because of its hardness and the high precision equired, so it is necessary to research the flying characteristic of the slider head in theory and to find out the relationship between the flying spacing of the burnishing head and its dimensional parameters, load, and load point position.

Generally the flying characters of the slider head are calculated by numerical solutions. This method needs a lot of calculation on a high-speed and largestorage computer.

The present paper adopts a simple approximate method. The static flying characteristic calculation of the burnishing head can be finished on an IBM-PC in a short time.

\section{Simple Approximate Calculation Method for the No Static Flying Characters of the Slider Head}

When the flying spacing is reduced to the submicron, the characteristic of the slider head can be governed by the following modified Reynolds equation by consideration of the molecular mean free path (MMEP).

$$
\begin{aligned}
& \frac{\partial}{\partial x}\left[\rho h^{3}\left(1+\frac{6 \lambda}{h}\right) \frac{\partial p}{\partial x}\right]+\frac{\partial}{\partial y}\left[\rho h^{3}\left(1+\frac{6 \lambda}{h}\right) \frac{\partial p}{\partial y}\right] \\
& \quad=6 \mu U \frac{\partial(\rho h)}{\partial x}+12 \mu \frac{\partial(\rho h)}{\partial t}
\end{aligned}
$$

where

$\rho=$ gas density

$h=$ flying spacing between disk surface and slider

$\lambda=$ molecular mean free path of gas

$\mu=$ viscosity coefficient of gas

$U=$ surface velocity

$p=$ gas pressure

$t=$ time

$x=$ coordinate along slider length direction

$y=$ coordinate along slider width direction as shown in Fig. 2.

Equation (1) is a nonlinear elliptic partial differential equation. It is extremely difficult to obtain an analytic solution. Generally, a numerical solution is used.

The process of the numerical solution is to divide the slider surface into a grid of meshes and the differential equation is replaced at each mesh point by a finite-difference equation at first. Then a set of nonlinear algebraic equations is obtained. The Newton-Raphson iteration method is applied to linearize them. Finally, the pressure data at each mesh can be calculated by solving the set of non-linear algebraic equations, original condition equations and boundary condition equations simultaneously. Further, we can calculate the buoyance and its center position by numerical integrating the pressure on the slider surface.

The calculation accuracy of this method depends on the number of meshes. The more meshes there are, the higher the accuracy is. This method needs a lot of computer memory and time. In view of this fact, this paper adopts a simple approximate calculation method instead of the above mentioned method.

A double-taperflat slider with a small width/ length ratio should follow the disk surface very well. So we have adopted this slider structure, shown in Fig. 3.

According to these structure features, a few assumptions can be made to satisfy simplifying needs.

1. Because $b / L<0.1$, the taper-flat slider can be considered as an infinitesimal width bearing. In this case, the first term on the left of Eq. (1) can be omitted.

2. The gas is assumed to be isothermal, so $p / p_{a}$ $=\rho / \rho_{a}=\lambda_{a} / \lambda$.

where

$p_{a}=$ ambient pressure

$\rho_{a}=$ ambient gas density

$\lambda_{a}=$ MMFP at ambient pressure

Because the load is small, the gas film density is assumed to be unchanged. Namely, gas compressibility is omitted.

3. The gas flow is assumed to be stable. Gas film thickness does not change with time. So, we omit

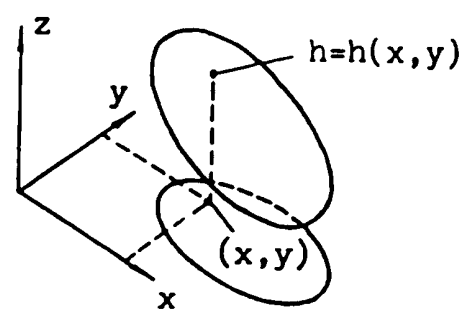

Fig. 2 The coordinate system of the lubricant region

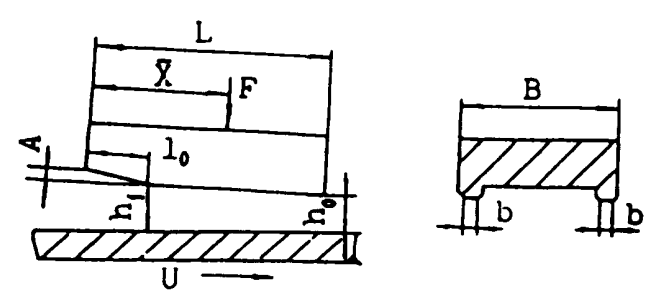

Fig. 3 Configuration of double-taper flat slider 
the time term in Eq. ( 1 ).

According to the three assumptions above, we can simplify Eq. (1) into the following form.

$$
\frac{\partial}{\partial y}\left(h^{3}\left(1+\frac{6 \lambda}{h}\right) \frac{\partial p}{\partial y}\right)=6 \mu U \frac{\partial h}{\partial x}
$$

Equation (2) can be written in a non-dimensional form as follows.

$$
\frac{\partial}{\partial Y}\left[H^{3}\left(1+\frac{6 M}{H}\right) \frac{\partial P}{\partial Y}\right]=\Lambda_{b} \frac{\partial H}{\partial X}
$$

where $H=h / h_{c}, Y=y / b, X=x / L, P=p / p_{a}, M=\lambda_{a} / h$,

$$
\Lambda_{b}=\frac{6 \mu U b^{2}}{p_{a} h_{0}^{2} L}
$$
found.

Equation ( 3 ) is the simplified equation to be

Boundary conditions are

$P=1$, at $X=0,1$ or $Y=0,1$

The formula of calculating the pressure distribution of the slider surface can be derived from Eqs. (3) and (4).

$$
P=1+\frac{\Lambda_{b}}{2} \frac{\partial H}{\partial X} \frac{Y(Y-1)}{\left(H^{3}+6 M H^{2}\right)}
$$

Figure 4 illustrates the pressure distribution on a double-taper flat slider surface.

The formula for calculating the buoyance can be derived by integrating the pressure on the slider surface.

$$
\begin{aligned}
F & =\frac{\mu U b^{3}}{6 M h_{0}^{2}}\left(1-\frac{1}{H_{1}+A / h_{0}}\right. \\
& \left.+\frac{1}{6 M} \ln \frac{6 M+H_{1}+A / h_{0}}{\left(H_{1}+A / h_{0}\right)(6 M+1)}\right)
\end{aligned}
$$

where $H_{1}=h_{1} / h_{0}$

The position of the buoyancy center in the $x$ direction can be calculated by the following equation.

$$
\begin{aligned}
\bar{X} & =\left\{1+\frac{L_{1}-1}{1-H_{1}}\left[\left(1+\frac{H_{1}-L_{1}}{\left(1-L_{1}\right) 6 M}\right) \ln \frac{6 M+H_{1}}{H_{1}(6 M+1)}\right]\right. \\
& +\frac{h_{0} L_{1}}{A}\left(1+\frac{H_{1}+A / h_{0}}{6 M}\right) \\
& \left.\times \ln \frac{\left(6 M+H_{1}+A / h_{0}\right) H_{1}}{\left(H_{1}+A / h_{0}\right)\left(6 M+H_{1}\right)}\right\} \\
& /\left[1-\frac{1}{H_{1}+A / h_{0}}+\frac{1}{6 M} \ln \frac{6 M+H_{1}+A / h_{0}}{\left(H_{1}+A / h_{0}\right)(6 M+1)}\right]
\end{aligned}
$$

When the slider head is flying above the disk surface, it should satisfy the equilibrium condition. Namely, the buoyancy is equal to the load, and the center of buoyancy coincides with the center of the load.

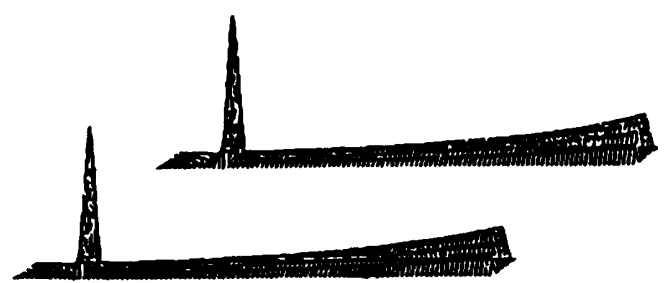

Fig. 4 Pressure distribution on a double-taper flat slider surface
According to the equilibrium condition and Eqs. (6) and (7), we programmed the calculation procedure for researching the characters of the slider head.

\section{Calculation Results for Burnishing Head}

Two kinds of burnishing heads have developed, one has a flying spacing of $0.5 \mu \mathrm{m}$, and the other a spacing of one is $0.2 \mu \mathrm{m}$.

Their dimensional parameters were determined by the above mentioned simple calculation procedure after a few time trial calculations, listed as follows.

1. $0.5 \mu \mathrm{m}$ flying spacing slider head $L=5 \mathrm{~mm}, l_{0}$ $=0.75 \mathrm{~mm}, b=0.46 \mathrm{~mm}, A=8 \mu \mathrm{m}, \bar{X}=0.53$;

2. $\quad 0.2 \mu \mathrm{m}$ flying spacing slider head $L=5 \mathrm{~mm}, l_{0}$ $=1 \mathrm{~mm}, b=0.25 \mathrm{~mm}, A=4 \mu \mathrm{m}, \bar{X}=0.53$.

Figure 5 shows the relation between the minimum flying spacing $\left(h_{0}\right)$ and the disk surface velocity $(U)$ at a series of loads $(F)$.

Figure 6 shows the relation between the minimum flying spacing $\left(h_{0}\right)$ and the slider width $(b)$ at a series of loads $(F)$.

Figure 7 shows the relation between the minimum flying spacing $\left(h_{0}\right)$ and the taper height $(A)$ at a series of loads $(F)$.

From Fig. 5, 6 and 7, we know that the slider

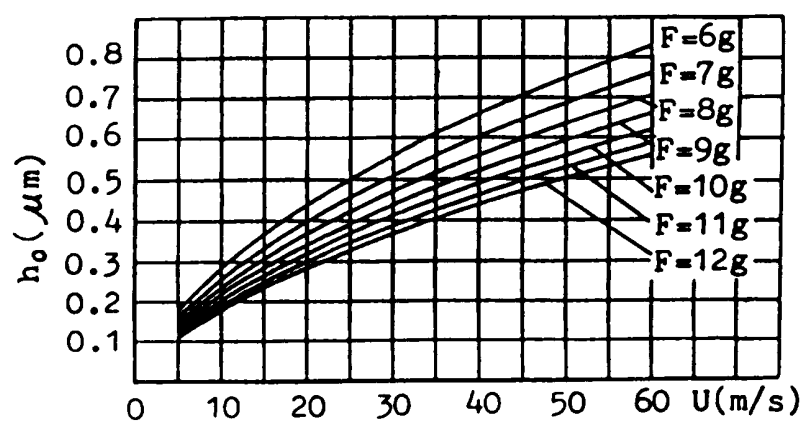

Fig. 5 Curve $h_{0}-U-F$ of $0.5 \mu \mathrm{m}$ flying spacing slider head

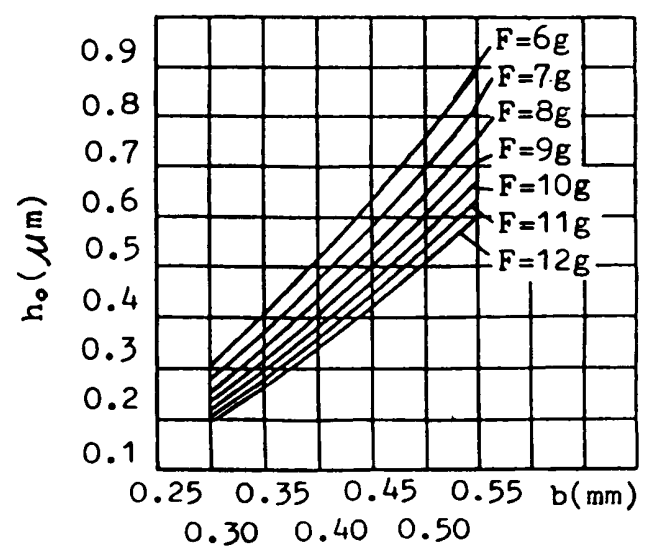

Fig. 6 Curve $h_{0}-b-F$ of $0.5 \mu \mathrm{m}$ flying spacing slider head $(U=40 \mathrm{~m} / \mathrm{s})$ 


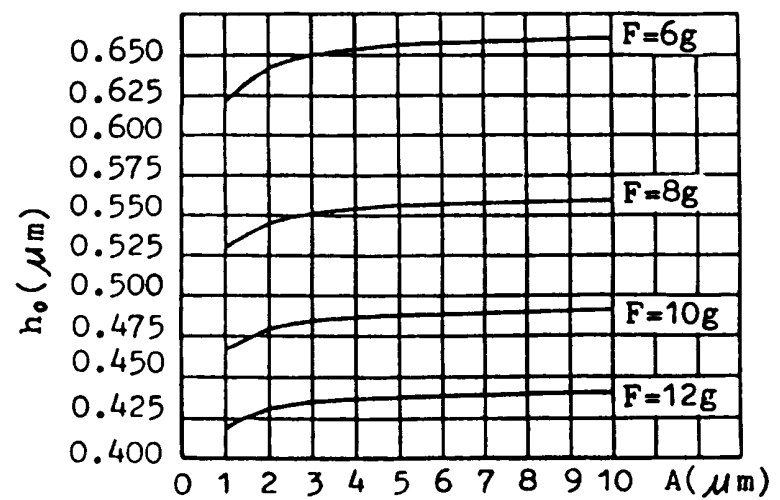

Fig. 7 Curve $h_{0}-A-F$ of $0.5 \mu \mathrm{m}$ flying spacing slider head $(U=40 \mathrm{~m} / \mathrm{s})$

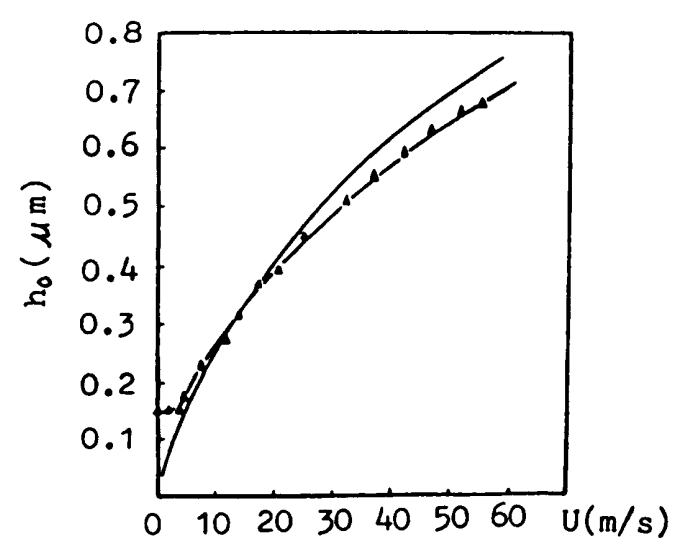

Fig. 8 Comparison between experimental data and calculated results

- simple approximate measurement

$-\Delta-\Delta$-real result of the measurement

width $(b)$ has the maximum influence on the flying spacing $\left(h_{0}\right)$ when the velocity of the magnetic disk $(U)$ is constant. The taper height $(A)$ has a great influence on the flying spacing $\left(h_{0}\right)$ when the taper height $(A)$ is very small, but the influence tends to decrease as the taper height $(A)$ increases. The taper length $l_{0}$ and the slider length $L$ have a small influence on the flying specing $\left(h_{0}\right)$.

\section{Experimental Research for Sapphire Burnishing Head}

We chose hard and transparent white sapphire as the material of burnishing head. After precision machining, double-taper flat burnishing heads were obtained. Dimensional parameters of one of the burnishing head are given below :

$$
\begin{aligned}
& L=5 \mathrm{~mm}, l_{0}=0.75 \mathrm{~mm}, b=0.46 \mathrm{~mm}, \\
& A=8 \mu \mathrm{m}, \bar{X}=0.53 .
\end{aligned}
$$

Because the burnishing head is transparent, the real flying spacing of the burnishing head can be measured by white light interference.

Figure 8 shows the experimental and calculated results.

By comparing the experimental data with the calculated results, we know that the experimental data valves are a little larger than the calculated results at low velocity and a little smaller at high velocity. Generally speaking, the experimental data are in good agreement with the calculated results. So we consider that the simple approximation calculation method to be useful in the design of burnishing heads.

\section{Conclusions}

The results can be summarized as follows:

1. The difference between the experimental data and calculated results enlarges as the disk surface velocity increases. This is due to the Omission of gas compressibility in the simple approximation calculation method. In fact, the gas density is different at different points in the flying spacing. The higher the disk surface velocity, the more obvious the compressibility effects.

2. The experimental data are slightly larger than the calculated results at low velocity. This is due to the omission of disk surface roughness effects in the calculation, the disk surface is considered as a ideal perfect plane.

3. The flying spacing does not decrease further if the disk surface velocity is very small. Because there are many little peaks on the disk surface and the minimum flying spacing $\left(h_{0}\right)$ can not be decreased if the slider surface is in contacts with the peaks.

4. Though there are some differences between the simple approximation calculation results and the experimental data. In the working velocity range, the difference value doesn't exceed $10 \%$. The simple approximation calculation is useful in the design of the burnishing head in the production of magnetic disk.

\section{References}

(1) Yan, zhi-yuan, Influence of Projections on the Coating Surface and its Solution, Electronic Com. puter, Vol. 1 (1984).

(2) Yao, jun-yuan, Zhang, yong-yi, Lin, cai-xin, Theory Calculation of Standard Colour Specification in the Measurement of White Light Interference, External Equipment of Electronic Computer, Vol. 2 (1983).

(3) Shen, Yang, Electronic Research Institute, Technology Measurement With Acoustic Emission, (1985).

(4) Mitsuya, Y., Kaneko, R., Molecular Mean Free Path Effects in Gas Lubricated Slider Bearing, Bull. JSME, Vol. 24, No. 187 (1981).

(5) Kaneko, R., Mitsuya, Y., Kogure, K., and Kita, T., Flying Heads With Sub-micro Spacing for Mag. netic Recording Disks, 8th Int. Gas Bearing Symp. (1981). 